\title{
On localised vibrations in incompressible pre-stressed transversely isotropic elastic solids
}

\author{
D. A. Prikazchikov, \\ Department of Theoretical and Applied Mechanics, \\ Russian State Open Technical University of Railway Transport \\ G. A. Rogerson* \\ Department of Mathematics \\ Keele University, Satffordshire, ST5 5BG \\ and \\ K. J. Sandiford, \\ Institute for Materials Research, \\ University of Salford, Salford. M5 4WT
}

October 25, 2006

\begin{abstract}
This paper is concerned with 2D localised vibration in incompressible pre-stressed fibre-reinforced elastic solids and the closely related problem of surface wave propagation in such materials. An appropriate constitutive model is derived and its stability discussed within the context of the strong ellipticity condition. Surface wave propagation in an associated half-space is considered, with the particular cases of propagation along a principal direction of primary deformation and that of almost inextensible fibres also investigated. The problems of free and forced edge vibration of a semiinfinite strip are analysed, revealing a link between the natural edge frequencies and the associated Rayleigh surface wave speed.
\end{abstract}

Key words: Vibration, pre-stress, elastic solid.

*Author for correspondence 


\section{Introduction}

The problem of localised dynamic phenomena in elastic bodies has a long history. It was Lord Rayleigh [1] who first considered the propagation of waves localised near the edge of an elastic half-space. Surface wave propagation has been extensively studied in various types of media, including pre-stressed elastic media, see e.g. Hayes and Rivlin [2], Chadwick and Jarvis [3], Dowaikh and Ogden [4]. The problem of edge vibration and edge resonance is relatively fresh compared to that of Rayleigh wave propagation. Shaw[5] was the first to observe the phenomena of edge vibrations in the case of a barium titanate disk. A considerable amount of effort resulted in a series of publications in this area, see e.g. Gazis and Mindlin [6], Gregory and Gladwell [7], Torvik [8], Roitberg et al. [9]. Our approach reveals a link between the edge eigenspectrum and the Rayleigh wave speed, seemingly first noted in the case of a semi-infinite cylindrical shell, see Kaplunov et al. [10]. Using this approach, two of the present authors have also previously contributed to the problems of $2 \mathrm{D}$ edge vibration of a pre-stressed isotropic elastic semi-strip and $3 \mathrm{D}$ edge waves in a semi-infinite plate, see Kaplunov et al. [11] and [12], respectively.

We remark that despite the previously mentioned research effort in this area, to date edge vibration modes have only been shown to exist for elastic materials with very specific types of boundary conditions. A full 3D treatment and a complete understanding of the precise class of boundary conditions which may support such modes remains beyond reach at this time. Additionally, for certain other types of material, for example piezo-elastic, questions relating to the uniqueness of surface waves are still unresolved. Bearing in mind the aforemention link between the edge eigenspectrum and the surface wave speed in the elastic case, extension of the treatment to other types of material offers significant challenge and leaves a number of open questions.

In the present paper we extend previous work to investigate the existence of edge vibration modes in a semi-infinite strip composed of a pre-stressed fibre-reinforced material. The corresponding constitutive model was seemingly first established by Prikazchikov and Rogerson [13]. We first obtain the propagation condition and make some remarks concerning stability within the context of the strong ellipticity condition. It is demonstrated that in the special case of the fibres being collinear with the principal axes the strong ellipticity conditions reduce to a form similar to the well-known form obtained by Ogden[14] for incompressible elastic solids. 
We then consider surface wave propagation in a 2D half-space, composed of an incompressible fibre-reinforced pre-stressed elastic material. The surface wave speed equation is derived, and a numerical analysis reveals its dependence on the normal Cauchy stress in the pre-stressed equilibrium state. The special case of the fibre direction lying along the principal in-plane direction is analysed, and it is shown that the surface wave speed equation then takes a form similar to that first obtained by Dowaikh and Ogden [4]. The case of almost inextensible fibres is also analysed.

We then proceed with an investigation of edge vibration of a semi-infinite strip. A link between the eigenspectrum and the surface wave speed is noted and numerical plots of the displacement eigenmodes are presented for both the free and forced vibration problems. The two special cases, namely the cases when the density of edge spectrum is increasing rapidly, and the case when the edge resonance phenomena is totally removed, are also studied with corresponding numerical illustrations provided. These scenarios are associated with the vanishing of the surface wave speed and degeneration into a body wave, respectively. Finally, we present results in respect of almost inextensible fibres.

The study is motivated by a number of considerations. Firstly, we aim to further elucidate the combined effects of pre-stress and material anisotropy on the existence of surface waves. Secondly, we highlight, for certain fibre orientation, that the frequencies of free edge vibration of a thin semi-infinite strip are functions of the corresponding Rayleigh surface wave speed. Finally, we demonstrate that knowledge of the influence of pre-stress on the existence of surface waves is enough to predict the degeneration of edge vibration as well as explicate various resonance phenomena. Understanding dynamic edge phenomena has potential applications in non-destructive evaluation. Moreover, there are a number of modern applications of thin structures, within which the ideas in this paper might offer some insight into the understanding the dynamic behaviour of thin structural components. A perhaps obvious example is aircraft wings, however in recent time the stability of ice sheets and artificial floating bodies such as floating runways, and the possible development of floating airports, have all received quite a bit of attention in the literature, see for example Oppenheimer [19], Inoue et al. [20] and Suzuki and Yoshida [21]. Although the boundary conditions associated with the last two examples are by nature more complex than those considered in this paper, the ideas within this paper hopefully point a way forward. 


\section{Governing equations}

We consider an incompressible transversely isotropic pre-stressed elastic material. The coordinate axes are chosen to coincide with the principal axes of primary deformation and the principal stretches are denoted by $\lambda_{1}, \lambda_{2}$ and $\lambda_{3}$, with the incompressibility constraint dictating that $\lambda_{1} \lambda_{2} \lambda_{3}=1$. In passing we remark that the squared stretches, $\lambda_{1}^{2}, \lambda_{2}^{2}$ and $\lambda_{3}^{2}$ are the eigenvalues of both the left and right Cauchy-Green strain tensors. In order to simplify the analysis, a plane-strain assumption will be adopted throughout this paper, with the displacement component $u_{2}=0$, and $u_{1}$ and $u_{3}$ being independent of $x_{2}$. The fibre direction $\mathbf{a}$ in the pre-stressed equilibrium state is orientated at an angle $\phi$ with $O x_{1}$ with the linearised equations of motion and the incompressibility condition presented as

$$
\begin{aligned}
B_{1111} u_{1,11}+2 B_{1131} u_{1,13}+B_{3131} u_{1,33}+B_{1113} u_{3,11}+\left(B_{1133}+B_{1331}\right) u_{3,13} & \\
& +B_{3133} u_{3,33}-p_{, 1}^{*}=\rho \ddot{u}_{1}, \\
B_{1113} u_{1,11}+\left(B_{1133}+B_{1331}\right) u_{1,13}+B_{3133} u_{1,33}+ & B_{1313} u_{3,11}+2 B_{1333} u_{3,13} \\
+ & B_{3333} u_{3,33}-p_{, 3}^{*}=\rho \ddot{u}_{3}, \\
u_{1,1}+u_{3,3}=0 &
\end{aligned}
$$

where $B_{i j k l}$ are components of the fourth order elasticity tensor, $\rho$ denotes the material density, and $p^{*}$ is the incremental pressure.

For the type of material considered we assume a general strain-energy function of the form

$$
W^{*}=W^{*}\left(I_{1}, I_{2}, I_{3}, I_{4}\right)+p(J-1)
$$

depending on the invariants

$$
I_{1}=C_{i i}, \quad I_{2}=\frac{1}{2}\left\{C_{i i} C_{j j}-C_{i j} C_{i j}\right\}, \quad I_{3}=a_{i} C_{i j} C_{j k} a_{k}, \quad I_{4}=a_{i} C_{i j} a_{j}, \quad J=\operatorname{det} \mathbf{F},
$$

where $\mathbf{F}$ is the gradient deformation tensor, $\mathbf{C}=\mathbf{F}^{\mathbf{T}} \mathbf{F}$ is the right Cauchy-Green tensor and $p$ is the pressure associated with the incompressibility constraint. We emphasise both that the strain energy function (2.2) is the most general form of strain energy associated with an incompressible, transversely isotropic elastic material and that we are working within a continuum framework. A tacit assumption of our continuum theory is that the fibres are assumed an inherent material property, rather than some form of inclusion. Moreover, within the continuum assumption the constitutive theory is an exact theory. The associated non-zero components of the fourth-order elasticity tensor, together with 
its definition, are given explicitly in Appendix A. For further details concerning either the continuum assumption, or the calculation of the tensor components, the reader is referred to Spencer [18].

The linearised incremental traction $\boldsymbol{\tau}^{(n)}(n=1,3)$ components associated with surface with the outward unit normal vector along $x_{1}$ and $x_{3}$ are given explicitly by

$$
\begin{aligned}
& \tau_{1}^{(1)}=\left(B_{1111}+\bar{p}\right) u_{1,1}+B_{1131} u_{1,3}+B_{1113} u_{3,1}+B_{1133} u_{3,3}-p^{*}, \\
& \tau_{3}^{(1)}=B_{1113} u_{1,1}+\left(B_{1331}+\bar{p}\right) u_{1,3}+B_{1313} u_{3,1}+B_{1333} u_{3,3}, \\
& \tau_{1}^{(3)}=B_{1131} u_{1,1}+B_{3131} u_{1,3}+\left(B_{1331}+\bar{p}\right) u_{3,1}+B_{3133} u_{3,3}, \\
& \tau_{3}^{(3)}=B_{1133} u_{1,1}+B_{3133} u_{1,3}+B_{3133} u_{1,3}+\left(B_{3333}+\bar{p}\right) u_{3,3}-p^{*} .
\end{aligned}
$$

\section{Propagation condition and stability}

We now proceed with an investigation of the propagation condition and stability within the framework of the strong ellipticity condition. This condition ensures real body wave speeds in all directions and is often taken as an indication of physically realistic dynamic response. Usually three waves propagate in any direction within an elastic solid. However, in the case of incompressibility this is reduced to two. The solutions for general wave propagation within the $\left(x_{1}, x_{3}\right)$-plane are given by

$$
\left(u_{1}, u_{3}, p^{*}\right)=(U, W, k P) e^{i k\left(x_{1} n_{1}+x_{3} n_{3}-v t\right)},
$$

where $\boldsymbol{n}=\left(n_{1}, 0, n_{3}\right)$ is an arbitrary unit vector in the $\left(x_{1}, x_{3}\right)$-plane. Substituting solutions (5) into the equations of motion and the incompressibility condition (1), we arrive at three homogeneous algebraic simultaneous equations in $U, W$ and $P$, which possess non-trivial solutions provided

$$
\bar{v}^{2}=\gamma n_{3}^{4}-2 \mu n_{1} n_{3}^{3}+2 \beta n_{1}^{2} n_{3}^{2}-2 \delta n_{1}^{3} n_{3}+\alpha n_{1}^{4}
$$

where

$$
\begin{gathered}
\bar{v}^{2}=\rho v^{2}, \quad \alpha=B_{1313}, \quad 2 \beta=B_{1111}+B_{3333}-2 B_{1133}-2 B_{1331}, \\
\gamma=B_{3131}, \quad \mu=B_{3133}-B_{1131}, \quad \delta=B_{1113}-B_{1333} .
\end{gathered}
$$

Thus, the strong ellipticity condition, ensuring real wave speeds in all directions, may be formulated as

$$
\gamma \chi^{4}+2 \mu \chi^{3}+2 \beta \chi^{2}+2 \delta \chi+\alpha>0, \quad(\forall \chi \in \mathcal{R})
$$


The condition (8) in general can hardly be simplified, though as we will show later it is sufficient to enable us to satisfy the decay conditions. However, in the two special cases of the fibre lying parallel or perpendicular to the direction of propagation, it reduces, due to the fact that $\mu=\delta=0$, to the well-known incompressible form, see Ogden [14],

$$
\alpha>0, \quad \gamma>0, \quad \beta+\sqrt{\alpha \gamma}>0 .
$$

\section{Surface wave propagation}

We now proceed to the problem of surface wave propagation in a semi-infinite plane composed of an incompressible pre-stressed transversely isotropic elastic material. The domain of the half-plane is given by $-\infty<x_{1}<\infty, 0 \leq x_{3}<\infty$. Solutions for the displacements and the pressure increment are sought in the form

$$
\left(u_{1}, u_{3}, p^{*}\right)=(U, W, k P) e^{i k\left(x_{1}-v t\right)} e^{-i k q x_{3}},
$$

where $\Im(q)<0$ ensures decay away from the boundary $x_{3}=0$.

By substituting (10) into the governing equations of motion and the incompressibility condition (1), we arrive at a linear homogeneous system of algebraic equations in respect of the constants $U, W$ and $P$. The condition that this system yields non-trivial solutions leads to the following equation for $q$

$$
\gamma q^{4}+2 \mu q^{3}+\left(2 \beta-\bar{v}^{2}\right) q^{2}+2 \delta q+\alpha-\bar{v}^{2}=0
$$

where $\alpha, \beta, \gamma, \delta$ and $\mu$ were defined in (7). We remark that the strong ellipticity condition (8) ensures that the roots of (11) corresponding to $\bar{v}^{2}=0$ are either imaginary or complex conjugates. As $v$ increases, the roots of (11) will remain complex until $\bar{v}=\bar{v}_{L}$, at which point at least one pair of roots coalesce and become real. The value of speed, $\bar{v}=\bar{v}_{L}$ say, is often referred to as the limiting speed and in general surface waves are only possible for $\bar{v}<\bar{v}_{L}$.

The displacements and the pressure increment may now be expressed as linear combi- 
nations of solutions decaying away from the edge $x_{3}=0$, namely

$$
\begin{aligned}
& u_{1}=\sum_{m=1}^{2} U_{m} e^{-i k q_{m} x_{3}} e^{i k\left(x_{1}-v t\right)}, \\
& u_{3}=\sum_{m=1}^{2} W_{m} e^{-i k q_{m} x_{3}} e^{i k\left(x_{1}-v t\right)}, \\
& p^{*}=\sum_{m=1}^{2} k P_{m} e^{-i k q_{m} x_{3}} e^{i k\left(x_{1}-v t\right),}
\end{aligned}
$$

where $U_{m}, W_{m}$ and $P_{m}(m=1,2)$ form a set of disposable constants, and $q_{1}$ and $q_{2}$ are the roots $(11)$ which satisfy the decay condition $\Im(q)<0$. Using the equations of motion and the incompressibility condition, solutions (12) may be presented in terms of two constants $W_{m}(m=1,2)$ as

$$
\begin{aligned}
& u_{1}=\sum_{m=1}^{2} q_{m} W_{m} e^{-i k q_{m} x_{3}} e^{i k\left(x_{1}-v t\right)}, \\
& u_{3}=\sum_{m=1}^{2} W_{m} e^{-i k q_{m} x_{3}} e^{i k\left(x_{1}-v t\right)}, \\
& p^{*}=\sum_{m=1}^{2} i k \mathcal{P}\left(q_{m}, \bar{v}^{2}\right) W_{m} e^{-i k q_{m} x_{3}} e^{i k\left(x_{1}-v t\right)},
\end{aligned}
$$

with $\mathcal{P}\left(q, \bar{v}^{2}\right)=B_{1113}+q\left(B_{1111}-B_{1133}-B_{1331}-\bar{v}^{2}\right)+q^{2}\left(B_{3133}-2 B_{1131}\right)+B_{3131} q^{3}$.

Substituting solutions (13) into the traction-free edge boundary conditions

$$
\tau_{1}^{(3)}=0, \quad \tau_{3}^{(3)}=0 \quad \text { at } x_{3}=0
$$

gives a homogeneous linear algebraic system in $W_{m}(m=1,2)$, namely

$$
\sum_{m=1}^{2} \mathcal{T}_{1}\left(q_{m}\right) W_{m}=0, \quad \quad \sum_{m=1}^{2} \mathcal{T}_{2}\left(q_{m}, \bar{v}^{2}\right) W_{m}=0,
$$

where

$$
\mathcal{T}_{1}(q)=\gamma q^{2}+\mu q-\left(\gamma-\sigma_{3}\right), \quad \mathcal{T}_{2}\left(q, \bar{v}^{2}\right)=\gamma q^{3}+2 \mu q^{2}+\left(\gamma-\sigma_{3}-\bar{v}^{2}+2 \beta\right) q+\delta,
$$

and $\sigma_{3}$ is the normal Cauchy stress in the pre-stressed equilibrium configuration, which is related to $\bar{p}$ through

$$
\sigma_{3}=B_{3131}-B_{1331}-\bar{p}
$$

see Ogden[14].

The surface wave speed equation is then obtained from (15) as a condition for the existence of non-trivial solutions for $W_{1}$ and $W_{2}$, namely

$$
\mathcal{T}_{1}\left(q_{1}\right) \mathcal{T}_{2}\left(q_{2}, \bar{v}^{2}\right)-\mathcal{T}_{1}\left(q_{2}\right) \mathcal{T}_{2}\left(q_{1}, \bar{v}^{2}\right)=0
$$


Equation (18) is in fact quadratic in $\sigma_{3}$, indicating that the surface wave speed crucially depends on the normal Cauchy stress. An illustration of a relation between $\bar{v}^{2}$ and $\sigma_{3}$ for various angles of the fibre direction is presented on Figure 1. For the purposes of numerical illustration, we assume the linear strain-energy function

$$
W^{*}=W_{1}^{*}\left(I_{1}-3\right)+W_{2}^{*}\left(I_{2}-3\right)+W_{3}^{*}\left(I_{3}-1\right)+W_{4}^{*}\left(I_{4}-1\right)+p(J-1),
$$

where $W_{i}^{*}(i=1, . ., 4)$ are the material parameters. As may well be expected, the curves

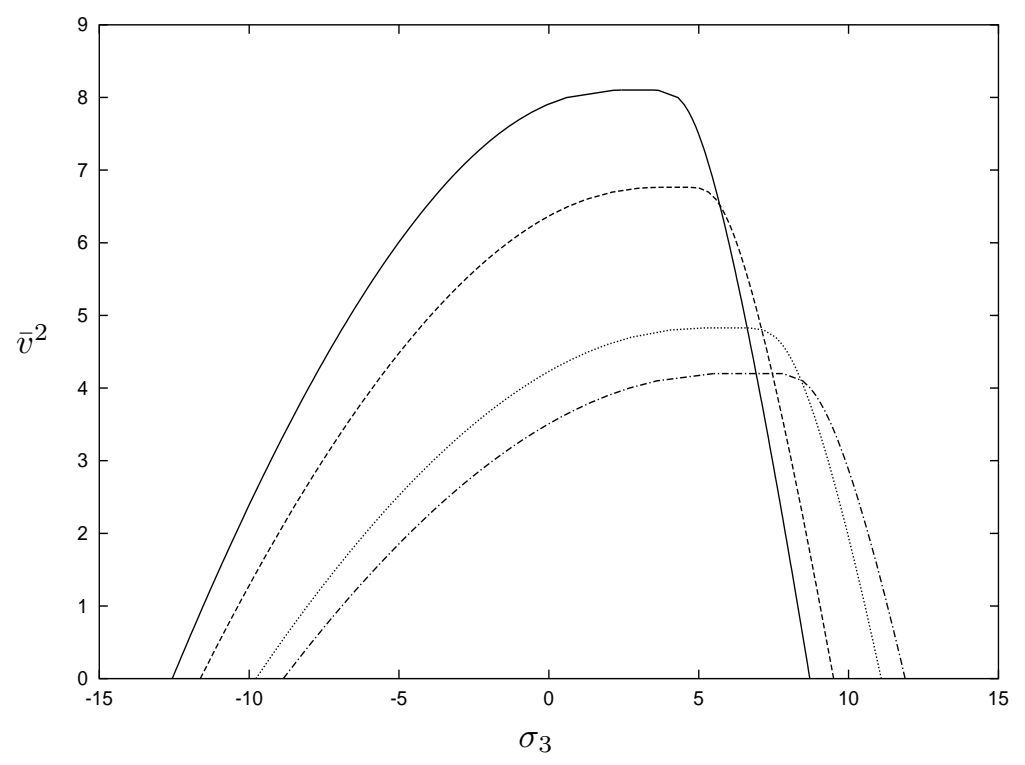

Figure 1: Surface wave speed against the normal Cauchy stress for the linear strain-energy function (19) with $\lambda_{1}=1.3, \lambda_{2}=0.7$ and $W_{1}^{*}=W_{2}^{*}=W_{3}^{*}=W_{4}^{*}=0.5: \phi=1^{0}$ (solid curve), $\phi=30^{\circ}$ (dashed curve), $60^{\circ}$ (dotted curve) and $89^{\circ}$ (dotted and dashed curve).

are generally of parabolic type, allowing real surface wave speed only within a certain interval of $\sigma_{3}$. Such curves are a generic feature of surface waves in pre-stressed elastic media.

\subsection{Special angles of the fibre.}

In this subsection we consider the two special cases of the fibres being along the principal directions, corresponding to $\phi=0$ and $\phi=\pi / 2$. As mentioned in the previous section, in this case the analysis simplifies significantly due to the fact that $\mu=\delta=0$. As a consequence, equation (11) is now a bi-quadratic in $q$, namely

$$
\gamma q^{4}+\left(2 \beta-\bar{v}^{2}\right) q^{2}+\alpha-\bar{v}^{2}=0
$$


from which we obtain

$$
\gamma\left(q_{1}^{2}+q_{2}^{2}\right)=\bar{v}^{2}-2 \beta, \quad \gamma q_{1}^{2} q_{2}^{2}=\alpha-\bar{v}^{2}
$$

Expressions for the incremental traction components also take simpler forms

$$
\begin{aligned}
& \tau_{1}^{(1)}=\left(B_{1111}+\bar{p}\right) u_{1,1}+B_{1133} u_{3,3}-p^{*}, \quad \tau_{3}^{(1)}=\left(B_{1331}+\bar{p}\right) u_{1,3}+B_{1313} u_{3,1}, \\
& \tau_{1}^{(3)}=B_{3131} u_{1,3}+\left(B_{1331}+\bar{p}\right) u_{3,1}, \quad \tau_{3}^{(3)}=B_{1133} u_{1,1}+\left(B_{3333}+\bar{p}\right) u_{3,3}-p^{*} .
\end{aligned}
$$

Substituting solutions (13) into the traction free boundary conditions (14) and following a procedure similar to that used in the general case, we obtain the surface wave speed equation

$$
\mathcal{T}_{1}\left(q_{1}\right) \mathcal{T}_{2}\left(q_{2}, \bar{v}^{2}\right)-\mathcal{T}_{1}\left(q_{2}\right) \mathcal{T}_{2}\left(q_{1}, \bar{v}^{2}\right)=0,
$$

where now

$$
\mathcal{T}_{1}(q)=\gamma q^{2}-\left(\gamma-\sigma_{3}\right), \quad \mathcal{T}_{2}\left(q, \bar{v}^{2}\right)=\gamma q^{3}+\left(\gamma-\sigma_{3}-\bar{v}^{2}+2 \beta\right) q
$$

Using the sum and product of the roots of the secular equation (20) which satisfy the decay condition, and introducing

$$
\gamma \eta^{2}=\alpha-\bar{v}^{2}
$$

equation (23) may now be transformed to the rather well-known form

$$
\eta^{3}+\eta^{2}+\left(\frac{2 \gamma+2 \beta-2 \sigma_{3}-\alpha}{\gamma}\right) \eta-\left(\frac{\gamma-\sigma_{3}}{\gamma}\right)^{2}=0,
$$

see Dowaikh and Ogden [4] for a full analysis of this equation. Equation (26) is formally identical to the one obtained in the cited paper and is shown there to have a unique positive root for $\eta$.

\subsection{Almost inextensible case}

Let us now consider an important special case when the the material is assumed to be almost inextensible along the fibre direction. An appropriate strain-energy function may then be introduced in the form

$$
W^{*}=W_{0}^{*}\left(I_{1}, I_{2}, I_{3}\right)+\frac{1}{8} E\left(I_{4}-1\right)^{2}-p(J-1) .
$$

In equation (27) the strain energy function is obtained by use of a Taylor series expansion appropriate to the case in which $\left(I_{4}-1\right)$ is small. It may be shown that nothing is gained 
by inclusion of a linear term in $\left(I_{4}-1\right)$ and that $E$ is an extensional modulus along the fibre direction, see Rogerson and Scott [15] and Scott [16]. We will assume that $E$ is much larger than any other material constant and is $O\left(\left(I_{4}-1\right)^{-1}\right)$, so that in the inextensible limit $E \rightarrow \infty$ and $\left(I_{4}-1\right) \rightarrow 0$ in such a way that the product $E\left(I_{4}-1\right) \rightarrow 2 T_{0}$, where $T_{0}$ is an arbitrary tension along the fibre direction.

The elasticity tensor may then be represented in the form

$$
B_{i j k l}=\tilde{B}_{i j k l}+T_{0} \delta_{j l} a_{i} a_{k}+E a_{i} a_{j} a_{k} a_{l}, \quad T_{0}=\frac{E\left(I_{4}-1\right)}{2},
$$

where tilde denotes dependence only on the three invariants $I_{1}, I_{2}, I_{3}$ with the non-zero elasticity tensor components given by

$$
\begin{aligned}
& B_{1111}=\tilde{B}_{1111}+T_{0} c_{\phi}^{2}+E c_{\phi}^{4}, \quad B_{1113}=\tilde{B}_{1113}+E c_{\phi}^{3} s_{\phi}, \quad B_{1131}=\tilde{B}_{1131}+T_{0} c_{\phi} s_{\phi}+E c_{\phi}^{3} s_{\phi}, \\
& B_{1133}=\tilde{B}_{1133}+E c_{\phi}^{2} s_{\phi}^{2}, \quad B_{1313}=\tilde{B}_{1313}+T_{0} c_{\phi}^{2}+E c_{\phi}^{2} s_{\phi}^{2}, \quad B_{1331}=\tilde{B}_{1331}+E c_{\phi}^{2} s_{\phi}^{2}, \\
& B_{1333}=\tilde{B}_{1333}+T_{0} c_{\phi} s_{\phi}+E c_{\phi} s_{\phi}^{3}, \quad B_{3131}=\tilde{B}_{3131}+T_{0} s_{\phi}^{2}+E c_{\phi}^{2} s_{\phi}^{2}, \\
& B_{3133}=\tilde{B}_{3133}+E s_{\phi}^{3} c_{\phi}, \quad B_{3333}=\tilde{B}_{3333}+T_{0} s_{\phi}^{2}+E s_{\phi}^{4} .
\end{aligned}
$$

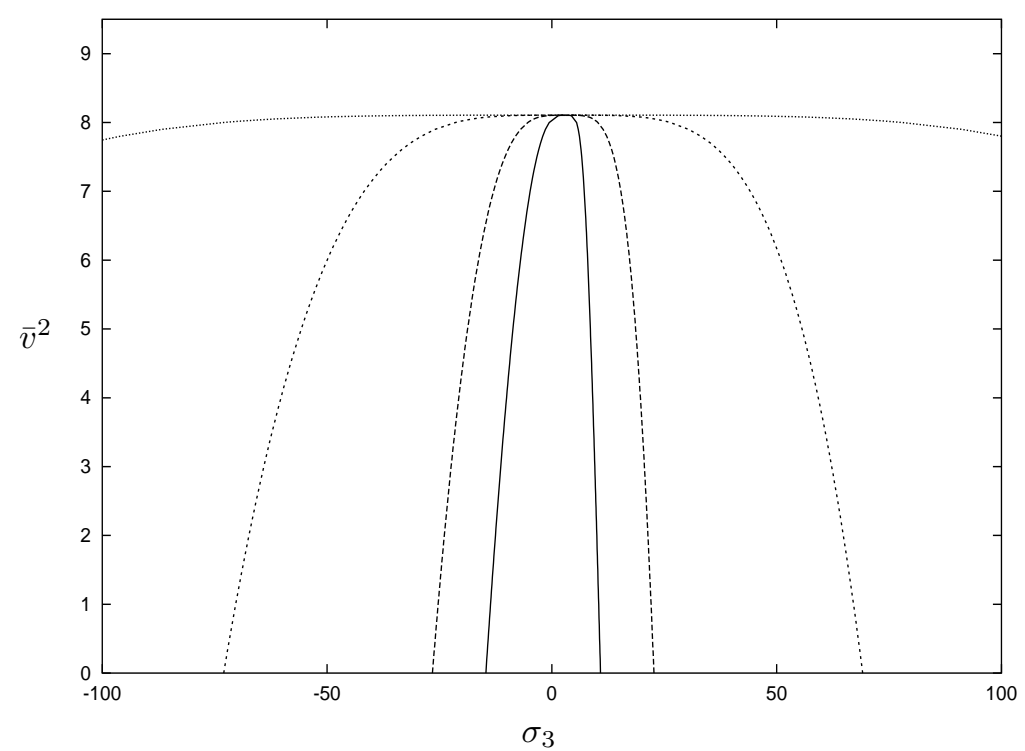

Figure 2: Surface wave speed against the normal Cauchy stress for the almost inextensible strain-energy function (27) with $\lambda_{1}=1.3, \lambda_{2}=0.7$ and $W_{1}^{*}=W_{2}^{*}=W_{3}^{*}=0.5$ for $\phi=1^{0}$ : $E=10.0$ (solid curve), $E=100.0$ (dashed curve), $E=1,000.0$ (bold dotted curve) and $E=10,000.0$ (dotted curve). 


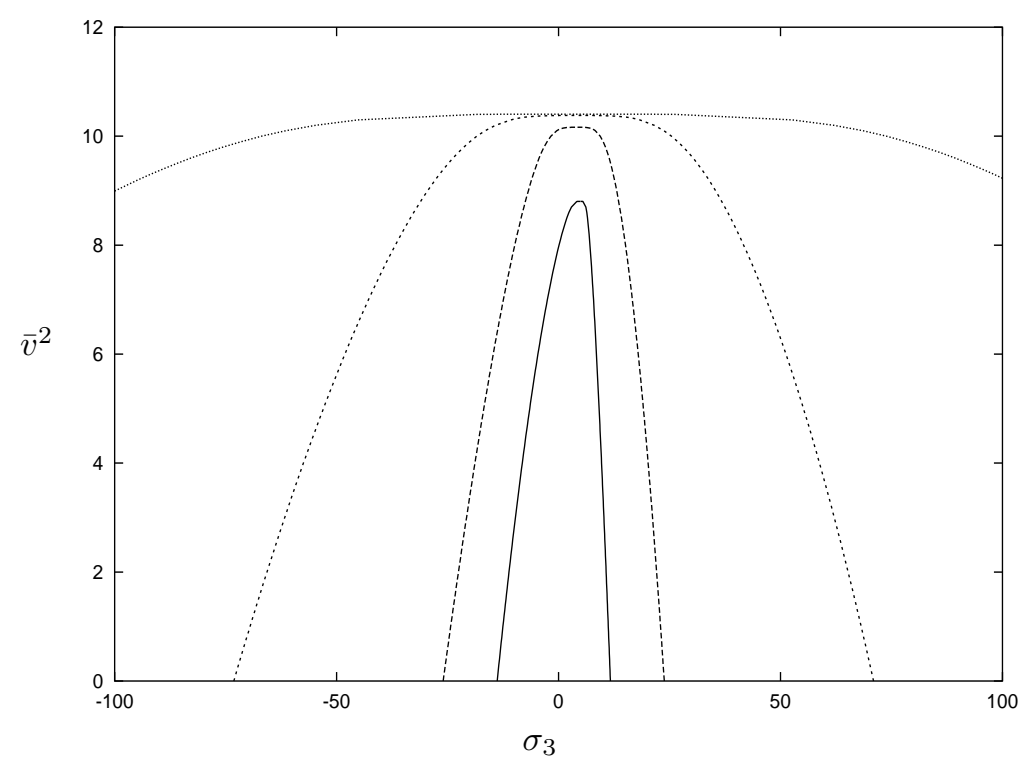

Figure 3: Surface wave speed against the normal Cauchy stress for the almost inextensible strain-energy function (27) with $\lambda_{1}=1.3, \lambda_{2}=0.7$ and $W_{1}^{*}=W_{2}^{*}=W_{3}^{*}=0.5$ for $\phi=30^{0}$ : $E=10.0$ (solid curve), $E=100.0$ (dashed curve), $E=1,000.0$ (bold dotted curve) and $E=10,000.0$ (dotted curve).

Some graphical illustrations of the surface wave speed for various angles of the fibre and several values of the extensional modulus are presented in Figures 2 and 3. It may be observed that Figure 2 demonstrates the typical type of behavior previously noted by Prikazchikov and Rogerson [17]. The curves of Figure 3, however, reveal some new features, namely that the point of degeneration of the surface wave speed into a body wave at the limiting speed $v_{L}$, the maximum speed, clearly depends on the value of the extensional modulus $E$. It is also worth remarking that the range of $\sigma_{3}$ within which a surface wave is possible increases as $E$ increases. However, as $E$ becomes large the surface wave degenerates into a body wave.

\section{$5 \quad$ Edge vibration of a semi-infinite strip}

This section is devoted to the study of free and forced edge vibrations of a semi-infinite strip subject to some mixed face boundary conditions. For simplicity, we assume that the fibres are parallel or perpendicular to the propagation direction. This will allow us to later decouple the problem and separate the transverse variable $x_{1}$. The domain occupied by 
the semi-strip is given by

$$
-h \leq x_{1} \leq h, \quad 0 \leq x_{3}<\infty .
$$

There are three types of mixed boundary conditions which may be imposed at the faces $x_{1}= \pm h$, for more details see Kaplunov et al. [11]. Here we will restrict our attention to one of those, namely to the case of a so-called "frictionless enclosure", in which the transverse displacements are precluded. This may be interpreted physically as the strip being surrounded by a stiff and frictionless enclosure. The face boundary conditions may then be written as

$$
u_{1}=\tau_{3}^{(1)}=0 \quad \text { at } x_{1}= \pm h .
$$

The other two cases of mixed boundary conditions given in Kaplunov et al. [11] may be treated similarly, however the details are not included in this paper.

\subsection{Free vibrations of a semi-infinite strip}

In the case of the free vibration problem, edge boundary conditions may be expressed as

$$
\tau_{1}^{(3)}=0, \quad \tau_{3}^{(3)}=0 \quad \text { at } x_{3}=0 .
$$

The governing equations of motion are taken in the form

$$
\begin{aligned}
& B_{1111} u_{1,11}+\left(B_{1133}+B_{1331}\right) u_{3,13}+B_{3131} u_{1,33}-p_{, 1}^{*}=\rho \ddot{u}_{1}, \\
& B_{1313} u_{3,11}+\left(B_{1133}+B_{1331}\right) u_{1,13}+B_{3333} u_{3,33}-p_{, 3}^{*}=\rho \ddot{u}_{3},
\end{aligned}
$$

which must be solved together with the linearised incompressibility condition

$$
u_{1,1}+u_{3,3}=0
$$

The solution for the displacements and pressure increment may be assumed to be in the form of two families

$$
\begin{aligned}
& \left(u_{1}, u_{3}, p^{*}\right)=\left(U \sin p_{1} x_{1}, W \cos p_{1} x_{1}, p_{1} P \cos p_{1} x_{1}\right) e^{-i q^{I} x_{3}} e^{i \omega t} \\
& \left(u_{1}, u_{3}, p^{*}\right)=\left(U \cos p_{2} x_{1}, W \sin p_{2} x_{1}, p_{2} P \sin p_{2} x_{1}\right) e^{-i q^{I I} x_{3}} e^{i \omega t} .
\end{aligned}
$$

We note that solutions (35) and (36) satisfy the mixed face boundary conditions (30) provided

$$
p_{1}=\frac{\Lambda_{s,(1)}^{n}}{h}, \quad \Lambda_{s,(1)}^{n}=n \pi, \quad p_{2}=\frac{\Lambda_{s,(2)}^{n}}{h}, \quad \Lambda_{s,(2)}^{n}=\frac{(2 n-1) \pi}{2}
$$


with $n=1,2,3, \ldots$.

We will consider the problem for the first family of solutions in detail and only present the main results for the second family. Substituting solutions (35) into the equations of motion and the incompressibility condition we arrive at a homogeneous system of equations in $U, W$ and $P$, from which it is possible to obtain the condition for this system to yield non-trivial solutions in the form

$$
\gamma\left(\hat{q}^{I}\right)^{4}+\left\{2 \beta-\hat{\Omega}_{1}^{2}\right\}\left(\hat{q}^{I}\right)^{2}+\left\{\alpha-\hat{\Omega}_{1}^{2}\right\}=0, \quad \hat{q}^{I}=\frac{q^{I} h}{\Lambda_{s,(1)}^{n}}, \quad \hat{\Omega}_{1}=\frac{\sqrt{\rho} \omega h}{\Lambda_{s,(1)}^{n}} .
$$

Using the equations of motion and the incompressibility condition, we may now present solutions for the displacements and the pressure increment as

$$
\begin{aligned}
u_{1} & =\left\{\sum_{k=1}^{2} i \hat{q}_{k}^{I} W^{(k)} e^{-i \hat{q}_{k}^{I} \Lambda_{s,(1)}^{n} \zeta}\right\} \sin \left(\Lambda_{s,(1)}^{n} \xi\right) e^{i \omega t}, \\
u_{3} & =\left\{\sum_{k=1}^{2} W^{(k)} e^{-i \hat{q}_{k}^{I} \Lambda_{s,(1)}^{n} \zeta}\right\} \cos \left(\Lambda_{s,(1)}^{n} \xi\right) e^{i \omega t}, \\
p^{*} & =\left\{\sum_{k=1}^{2} i p_{1} \hat{q}_{k}^{I} W^{(k)} \mathcal{H}\left(\hat{q}_{k}^{I}, \Omega_{1}^{2}\right) e^{-i \hat{q}_{k}^{I} \Lambda_{s,(1)}^{n} \zeta}\right\} \cos \left(\Lambda_{s,(1)}^{n} \xi\right) e^{i \omega t},
\end{aligned}
$$

where $\hat{q}_{k}^{I} \quad(k=1,2)$ are the two roots of the secular equation (38), which satisfy the decay condition $\Im(q)<0$ and

$$
x_{1}=h \xi, \quad x_{3}=h \zeta, \quad \mathcal{H}\left(\hat{q}, \hat{\Omega}^{2}\right)=\hat{q}^{2} B_{3131}+\left(B_{1111}-B_{1133}-B_{1331}\right)-\hat{\Omega}^{2} .
$$

Substituting solutions (39) into the traction free edge boundary conditions (31), we arrive at a homogeneous system in $W^{(1)}$ and $W^{(2)}$, namely

$$
\sum_{k=1}^{2} \mathcal{T}_{1}\left(\hat{q}_{k}^{I}\right) W^{(k)}=0, \quad \sum_{k=1}^{2} \mathcal{T}_{2}\left(\hat{q}_{k}^{I}, \hat{\Omega}_{1}^{2}\right) W^{(k)}=0,
$$

where the functions $\mathcal{T}_{1}(q)$ and $\mathcal{T}_{2}\left(q, \Omega^{2}\right)$ were defined in $(24)$. The condition that this system possesses non-trivial solutions may be written as

$$
\mathcal{S}_{1 s}\left(\hat{q}_{1}^{I}, \hat{q}_{2}^{I}, \hat{\Omega}_{1}^{2}\right) \equiv \mathcal{T}_{1}\left(\hat{q}_{1}^{I}\right) \mathcal{T}_{2}\left(\hat{q}_{2}^{I}, \hat{\Omega}_{1}^{2}\right)-\mathcal{T}_{1}\left(\hat{q}_{2}^{I}\right) \mathcal{T}_{2}\left(\hat{q}_{1}^{I}, \hat{\Omega}_{1}^{2}\right)=0
$$

Using the sum and product of the roots of the secular equation (38), taking note of the decay condition and introducing

$$
\eta=\sqrt{\frac{\alpha-\hat{\Omega}_{1}^{2}}{\gamma}}
$$


it is possible to rewrite $(42)$ as

$$
\eta^{3}+\eta^{2}+\left(\frac{2 \gamma+2 \beta-2 \sigma_{3}-\alpha}{\gamma}\right) \eta-\left(\frac{\gamma-\sigma_{3}}{\gamma}\right)^{2}=0,
$$

which is precisely equation (26), possessing a unique positive solution for $\eta$, see Dowaikh and Ogden [4].

Thus, we have established a link between the edge spectrum of the semi-strip with the speed of the Rayleigh-type wave localised near the edge $x_{3}=0$, namely

$$
\Omega_{1}^{(n)}=\frac{\Lambda_{s,(1)}^{n} c_{R}}{h}
$$

where $c_{R}$ denotes the Rayleigh wave speed value. The corresponding eigenfunctions may be obtained as

$$
\begin{aligned}
& u_{1}=\left\{\mathcal{T}_{2}\left(\hat{q}_{2}^{I}, \hat{\Omega}_{1}^{2}\right) i \hat{q}_{1}^{I} e^{-i \hat{q}_{1}^{I} \Lambda_{s,(1)}^{n} \zeta}-\mathcal{T}_{2}\left(\hat{q}_{1}^{I}, \hat{\Omega}_{1}^{2}\right) i \hat{q}_{2}^{I} e^{-i \hat{q}_{2}^{I} \Lambda_{s,(1)}^{n} \zeta}\right\} \sin \left(\Lambda_{s,(1)}^{n} \xi\right) \tilde{W}_{0}, \\
& u_{3}=\left\{\mathcal{T}_{2}\left(\hat{q}_{2}^{I}, \hat{\Omega}_{1}^{2}\right) e^{-i \hat{q}_{1}^{I} \Lambda_{s,(1)}^{n} \zeta}-\mathcal{T}_{2}\left(\hat{q}_{1}^{I}, \hat{\Omega}_{1}^{2}\right) e^{-i \hat{q}_{2}^{I} \Lambda_{s,(1)}^{n} \zeta}\right\} \cos \left(\Lambda_{s,(1)}^{n} \xi\right) \tilde{W}_{0}, \\
& p^{*}=i p_{1}\left\{-\mathcal{T}_{2}\left(\hat{q}_{2}^{I}, \hat{\Omega}_{1}^{2}\right) \mathcal{H}\left(\hat{q}_{1}^{I}, \hat{\Omega}_{1}^{2}\right) \hat{q}_{1}^{I} e^{-i \hat{q}_{1}^{I} \Lambda_{s,(1)}^{n} \zeta}+\mathcal{T}_{2}\left(\hat{q}_{1}^{I}, \hat{\Omega}_{1}^{2}\right) \mathcal{H}\left(\hat{q}_{2}^{I}, \hat{\Omega}_{1}^{2}\right) \hat{q}_{2}^{I} e^{i-\hat{q}_{2}^{I} \Lambda_{s,(1)}^{n} \zeta}\right\} \times \\
& \cos \left(\Lambda_{s,(1)}^{n} \xi\right) \tilde{W}_{0},
\end{aligned}
$$

where the variation in time has been included in $\tilde{W}_{0}$. Some typical graphical illustrations, depicting the displacement eigenmodes decaying away from the edge $x_{3}=0$, are presented in Figure 4. The dependence of the transverse displacement eigenmodes on the longitudinal variable $x_{3}$ is shown on Figure 4(a). It may be observed that the displacement is localised near the edge $x_{3}=0$ and decays rapidly away from the edge. It is also clear from (46) that it decreases more rapidly as the mode number $n$ increases. Similar features may be observed for the longitudinal displacement presented in Figure 4(b).

Analogous results can be obtained for the second family of solutions (36). The solutions for the displacements and the pressure increment may first be obtained as

$$
\begin{aligned}
& u_{1}=\left\{\sum_{k=1}^{2}-i \hat{q}_{k}^{I I} W^{(k)} e^{-i \hat{q}_{k}^{I I} \Lambda_{s,(2)}^{n} \zeta}\right\} \cos \left(\Lambda_{s,(2)}^{n} \xi\right) e^{i \omega t}, \\
& u_{3}=\left\{\sum_{k=1}^{2} W^{(k)} e^{-i \hat{q}_{k}^{I I} \Lambda_{s,(2)}^{n} \zeta}\right\} \sin \left(\Lambda_{s,(2)}^{n} \xi\right) e^{i \omega t}, \\
& p^{*}=\left\{\sum_{k=1}^{2} i p_{2} \hat{q}_{k}^{I I} W^{(k)} \mathcal{H}\left(\hat{q}_{k}^{I I}, \hat{\Omega}_{2}^{2}\right) e^{-i \hat{q}_{k}^{I I} \Lambda_{s,(2)}^{n} \zeta}\right\} \sin \left(\Lambda_{s,(2)}^{n} \xi\right) e^{i \omega t},
\end{aligned}
$$




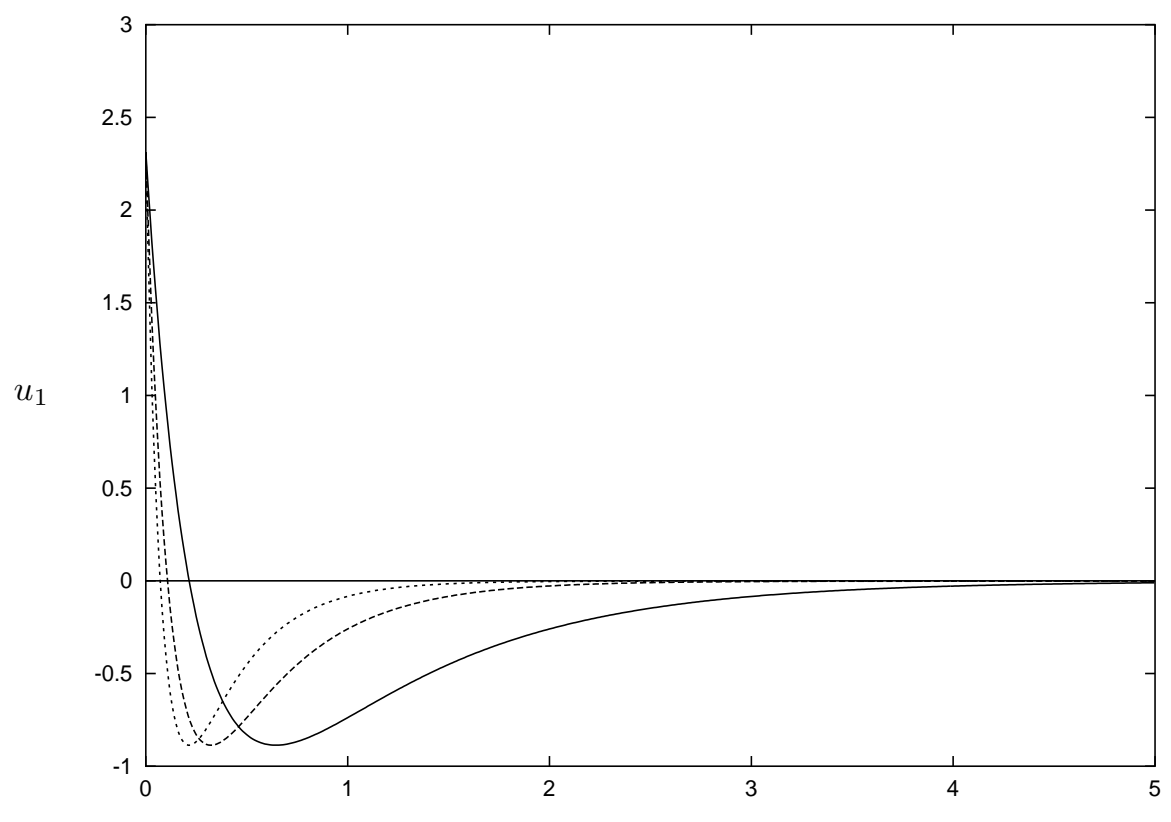

(a) $\quad x_{3}$

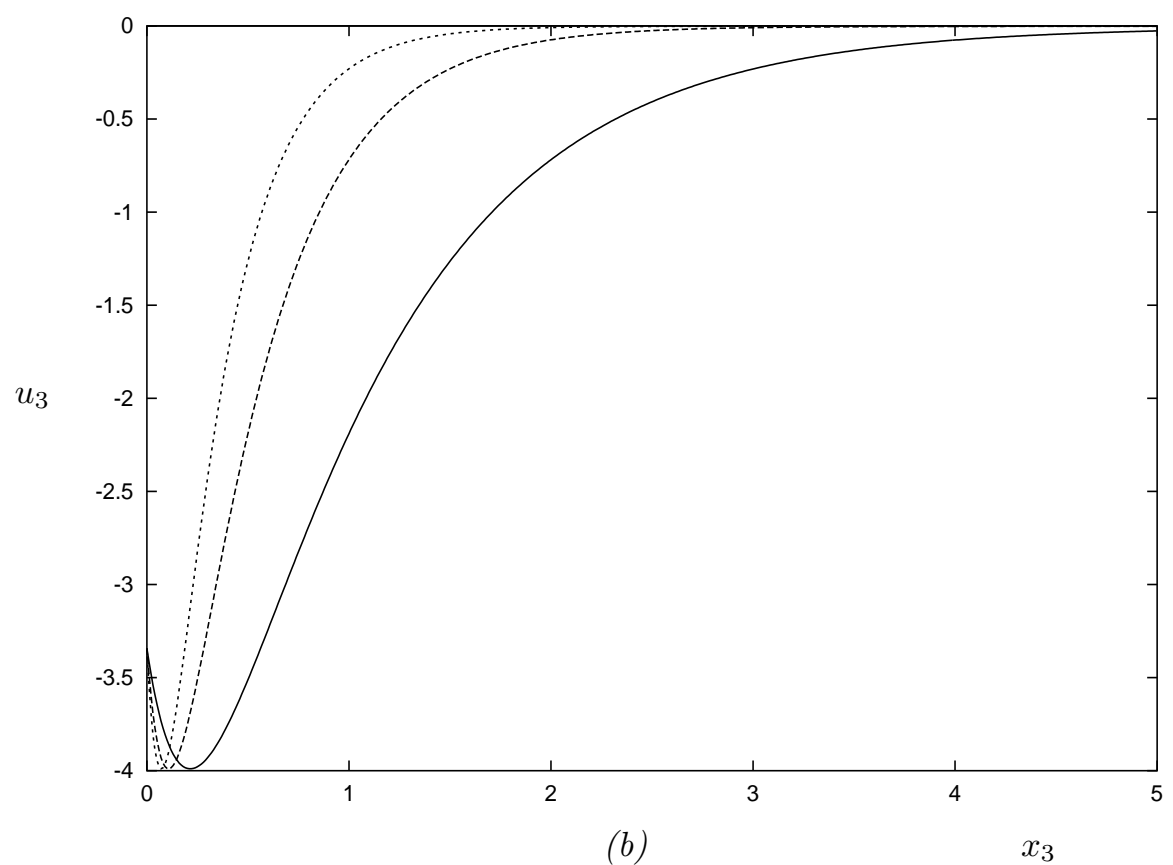

Figure 4: (a) The imaginary part of $u_{1}$, showing decay away from the edge in respect of the linear strain-energy function (19) with $\lambda_{1}=1.3, \lambda_{2}=0.7$ and $W_{1}^{*}=W_{2}^{*}=W_{3}^{*}=$ $W_{4}^{*}=0.5$ : First mode (solid curve), second mode (dashed curve), third mode (dotted curve).

(b) The imaginary part of $u_{2}$, showing decay away from the edge in respect of the linear strain-energy function (19) with $\lambda_{1}=1.3, \lambda_{2}=0.7$ and $W_{1}^{*}=W_{2}^{*}=W_{3}^{*}=W_{4}^{*}=0.5$ : First mode (solid curve), second mode (dashed curve), third mode (dotted curve). 
where $\hat{q}_{k}^{I I} \quad(k=1,2)$ are the decaying roots of

$$
\gamma\left(\hat{q}^{I I}\right)^{4}+\left\{2 \beta-\hat{\Omega}_{2}^{2}\right\}\left(\hat{q}^{I I}\right)^{2}+\left\{\alpha-\hat{\Omega}_{2}^{2}\right\}=0, \quad \hat{q}^{I I}=\frac{q^{I I} h}{\Lambda_{s,(2)}^{n}}, \quad \hat{\Omega}_{2}=\frac{\sqrt{\rho} \omega h}{\Lambda_{s,(2)}^{n}},
$$

with $\xi, \zeta$ and $\mathcal{H}\left(\hat{q}, \hat{\Omega}^{2}\right)$ defined in (40).

Substituting solutions (47) into the traction free edge boundary conditions (31), we arrive at a homogeneous system in $W^{(1)}$ and $W^{(2)}$

$$
\sum_{k=1}^{2} \mathcal{T}_{1}\left(\hat{q}_{k}^{I I}\right) W^{(k)}=0, \quad \sum_{k=1}^{2} \mathcal{T}_{2}\left(\hat{q}_{k}^{I I}, \hat{\Omega}_{2}^{2}\right) W^{(k)}=0
$$

from which we obtain the edge wave speed equation as a condition of this system possessing non-trivial solutions

$$
\mathcal{S}_{2 s}\left(\hat{q}_{1}^{I I}, \hat{q}_{2}^{I I}, \hat{\Omega}_{2}^{2}\right) \equiv \mathcal{T}_{1}\left(\hat{q}_{1}^{I I}\right) \mathcal{T}_{2}\left(\hat{q}_{2}^{I I}, \hat{\Omega}_{2}^{2}\right)-\mathcal{T}_{1}\left(\hat{q}_{2}^{I I}\right) \mathcal{T}_{2}\left(\hat{q}_{1}^{I I}, \hat{\Omega}_{2}^{2}\right)=0
$$

After some small algebraic manipulations it can be presented as

$$
\eta^{3}+\eta^{2}+\left(\frac{2 \gamma+2 \beta-2 \sigma_{3}-\alpha}{\gamma}\right) \eta-\left(\frac{\gamma-\sigma_{3}}{\gamma}\right)^{2}=0
$$

where

$$
\eta^{2}=\frac{\alpha-\hat{\Omega}_{2}^{2}}{\gamma}
$$

Thus, once again a link between the edge spectrum of the semi-strip with the speed of the associated Rayleigh-type wave may be established in the form

$$
\Omega_{2}^{(n)}=\frac{\Lambda_{s,(2)}^{n} c_{R}}{h} .
$$

The corresponding eigenfunctions may be obtained as

$$
\begin{gathered}
u_{1}=\left\{-\mathcal{T}_{2}\left(\hat{q}_{2}^{I I}, \hat{\Omega}_{2}^{2}\right) i \hat{q}_{1}^{I I} e^{-i \hat{q}_{1}^{I I} \Lambda_{s,(2)}^{n} \zeta}+\mathcal{T}_{2}\left(\hat{q}_{1}^{I I}, \hat{\Omega}_{2}^{2}\right) i \hat{q}_{2}^{I I} e^{-i \hat{q}_{2}^{I I} \Lambda_{s,(2)}^{n} \zeta}\right\} \cos \left(\Lambda_{s,(2)}^{n} \xi\right) \tilde{W}_{0}, \\
u_{3}=\left\{\mathcal{T}_{2}\left(\hat{q}_{2}^{I I}, \hat{\Omega}_{2}^{2}\right) e^{-i \hat{q}_{1}^{I I} \Lambda_{s,(2)}^{n} \zeta}-\mathcal{T}_{2}\left(\hat{q}_{1}^{I I}, \hat{\Omega}_{2}^{2}\right) e^{-i \hat{q}_{2}^{I I} \Lambda_{s,(2)}^{n} \zeta}\right\} \sin \left(\Lambda_{s,(2)}^{n} \xi\right) \tilde{W}_{0}, \\
p^{*}=i p_{2}\left\{-\mathcal{T}_{2}\left(\hat{q}_{2}^{I I}, \hat{\Omega}_{2}^{2}\right) \mathcal{H}\left(\hat{q}_{1}^{I I}, \hat{\Omega}_{2}^{2}\right) \hat{q}_{1}^{I I} e^{-i \hat{q}_{1}^{I I} \Lambda_{s,(2)}^{n} \zeta}+\mathcal{T}_{2}\left(\hat{q}_{1}^{I I}, \hat{\Omega}_{2}^{2}\right) \mathcal{H}\left(\hat{q}_{2}^{I I}, \hat{\Omega}_{2}^{2}\right) \hat{q}_{2}^{I I} e^{-i \hat{q}_{2}^{I I} \Lambda_{s,(2)}^{n} \zeta}\right\} \\
\times \sin \left(\Lambda_{s,(2)}^{n} \xi\right) \tilde{W}_{0},
\end{gathered}
$$

where the variation in time has been included in $\tilde{W}_{0}$. 


\subsection{Forced vibration of a semi-infinite strip}

We now consider the problem of forced vibration. In the case of the first family of solutions (35), illustrative edge boundary conditions are taken in the form

$$
\begin{gathered}
f\left(x_{1}\right)=\left.\tau_{1}^{(3)}\right|_{x_{3}=0}=0 \\
g\left(x_{1}\right)=\left.\tau_{3}^{(3)}\right|_{x_{3}=0}=\sigma_{0}\left(\frac{1}{3}-\frac{x_{1}^{2}}{h^{2}}\right) e^{i \omega t} \quad \text { or } \quad \tilde{g}(\xi)=\sigma_{0}\left(\frac{1}{3}-\xi^{2}\right) e^{i \omega t} .
\end{gathered}
$$

This type of loading allows $\tilde{g}(\xi)$ to be expanded as a Fourier series in respect of the eigenfunctions, namely

$$
\tilde{g}(\xi)=\frac{4 \sigma_{0}}{\pi^{2}} \sum_{n=1}^{\infty} \frac{(-1)^{n+1} \cos \left(\Lambda_{s,(1)}^{n} \xi\right)}{n^{2}} e^{i \omega t} .
$$

Using (39), it is now possible to represent the boundary conditions as

$$
\begin{aligned}
& \sum_{n=1}^{\infty}\left\{\mathcal{T}_{1}\left(\hat{q}_{1}^{I}\right) W_{n}^{(1)}+\mathcal{T}_{1}\left(\hat{q}_{2}^{I}\right) W_{n}^{(2)}\right\}=0 \\
& \sum_{n=1}^{\infty}\left\{\mathcal{T}_{2}\left(\hat{q}_{1}^{I}, \hat{\Omega}_{1}^{2}\right) W_{n}^{(1)}+\mathcal{T}_{2}\left(\hat{q}_{1}^{I}, \hat{\Omega}_{1}^{2}\right) W_{n}^{(2)}\right\}=4 \sigma_{0} h \sum_{n=1}^{\infty} \frac{(-1)^{n+1}}{\left[\Lambda_{s,(1)}^{n}\right]^{3}} .
\end{aligned}
$$

From these two equations it may be deduced that

$$
W_{n}^{(1)}=\frac{4 i \sigma_{0} h \mathcal{T}_{1}\left(\hat{q}_{2}^{I}\right)(-1)^{n+1}}{\left[\Lambda_{s,(1)}^{n}\right]^{3} \mathcal{S}_{1 s}\left(\hat{q}_{1}^{I}, \hat{q}_{2}^{I}, \hat{\Omega}_{1}^{2}\right)}, \quad W_{n}^{(2)}=\frac{4 i \sigma_{0} h \mathcal{T}_{1}\left(\hat{q}_{1}^{I}\right)(-1)^{n}}{\left[\Lambda_{s,(1)}^{n}\right]^{3} \mathcal{S}_{1 s}\left(\hat{q}_{1}^{I}, \hat{q}_{2}^{I}, \hat{\Omega}_{1}^{2}\right)} .
$$

Appropriate solutions for the displacement components and pressure increment associated with the $n^{\text {th }}$ harmonic are provided by

$$
\begin{aligned}
& u_{1}^{(n)}=\left\{\sum_{m=1}^{2} i \hat{q}_{m}^{I} W_{n}^{(m)} e^{-i \hat{q}_{m}^{I} \Lambda_{s,(1)}^{n} \zeta} \sin \left(\Lambda_{s,(1)}^{n} \xi\right) e^{i \omega t}\right. \\
& u_{3}^{(n)}=\left\{\sum_{m=1}^{2} W_{n}^{(m)} e^{-i \hat{q}_{m}^{I} \Lambda_{s,(1)}^{n} \zeta}\right\} \cos \left(\Lambda_{s,(1)}^{n} \xi\right) e^{i \omega t} \\
& p^{*(n)}=\left\{\sum_{m=1}^{2}-i p_{1} \hat{q}_{m}^{I} W_{n}^{(m)} \mathcal{H}\left(\hat{q}_{m}^{I}, \hat{\Omega}_{1}^{2}\right) e^{-i \hat{q}_{m}^{I} \Lambda_{s,(1)}^{n} \zeta}\right\} \cos \left(\Lambda_{s,(1)}^{n} \xi\right) e^{i \omega t} .
\end{aligned}
$$

Kaplunov et al.[11], in the context of an incompressible pre-stressed isotropic elastic semi-strip, have shown that pre-stress may crucially effect the edge resonance phenomena. Here we will also demonstrate this phenomena in the case of a strip composed of a fibre-reinforced pre-stressed elastic material. It is known that a surface wave propagates provided the normal Cauchy stress $\sigma_{3}$ is in the interval of

$$
\sigma_{3}=\gamma-\sqrt{\gamma \alpha}+d \sqrt{2 \sqrt{\gamma \alpha}(\beta+\sqrt{\gamma \alpha})}, \quad d \in[-1 . .1],
$$



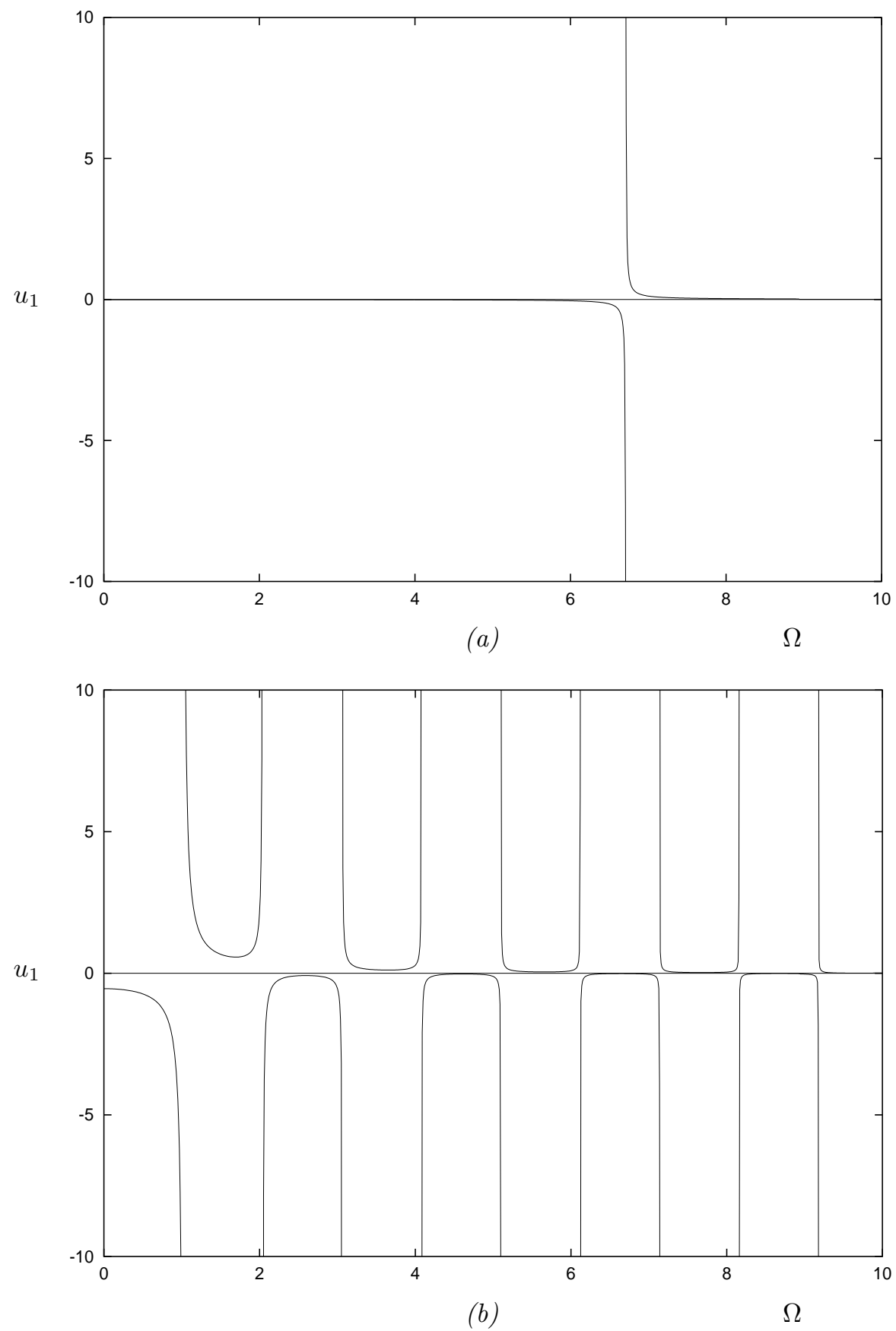

Figure 5: (a) The displacement $u_{1}$ as the surface wave speed tends to zero for the linear strain-energy function (19) with $\lambda_{1}=1.3, \lambda_{2}=0.7$ and $W_{1}^{*}=W_{2}^{*}=W_{3}^{*}=W_{4}^{*}=0.5$ and $d=-0.5$,

(b) The displacement $u_{1}$ as the surface wave speed tends to zero for the linear strain-energy function (19) with $\lambda_{1}=1.3, \lambda_{2}=0.7$ and $W_{1}^{*}=W_{2}^{*}=W_{3}^{*}=W_{4}^{*}=0.5$ and $d=-0.95$. 
see Dowaikh and Ogden [4]. Furthermore, the ends of the stability interval, where $d=1$ and $d=-1$, provide a standing wave. In the case when the surface wave speed tends to zero, the phenomena of edge spectrum density increase may be observed. A clear confirmation of this may be noticed from Figure 5. Figures 5(a) and (b) are plotted for different values of normal Cauchy stress such that the surface wave speed tends to zero. Figure 5(a) corresponds to $d=-0.5$ and Figure $5(\mathrm{~b})$ is produced for $d=-0.95$. It may be observed that as $d \rightarrow-1$ the density of resonances increases and in the limit they will all merge into zero.

Another possible feature of pre-stress is that the surface wave may degenerate into a body wave. It is known from Dowaikh and Ogden [4] that the value of $\sigma_{3}=\gamma$ corresponds to degeneration of the surface wave into a body wave. The four displacement modes presented on Figure 6 are calculated for $\sigma_{3}=\gamma+\Delta$ with $\Delta$ tending to zero from (a) to (d). It is anticipated that the amplitude of the resonances should vanish as the body wave speed is approached, and this is readily observed from Figure 6. One point worth remarking in respect of Figures 5 and 6 is the applicability of the linearised theory when such large resonance-type displacements are present. Such a theory, based upon a small amplitude assumption, is not able to accurately determine stresses and other field quantities within, or close to, resonance-type states. It is however, perfectly capable of establishing the set of circumstances when this type of phenomena will occur. Moreover, our intention in presenting these figures is to demonstrate the role played by pre-stress in the on-set or removal of such potential resonance-type phenomena.

Similar results may readily be obtained for the second family of solutions (5.8). We now suppose

$$
\begin{gathered}
f\left(x_{1}\right)=\left.\tau_{1}^{(3)}\right|_{x_{3}=0}=\sigma_{0}\left(\frac{1}{3}-\frac{x_{1}^{2}}{h^{2}}\right) e^{i \omega t} \text { or } \tilde{f}(\xi)=\sigma_{0}\left(\frac{1}{3}-\xi^{2}\right) e^{i \omega t}, \\
g\left(x_{1}\right)=\left.\tau_{3}^{(3)}\right|_{x_{3}=0}=0 .
\end{gathered}
$$

The function $f\left(x_{1}\right)$ may now be expanded as a Fourier series to reveal

$$
\tilde{f}(\xi)=\frac{32 \sigma_{0}}{\pi^{3}} \sum_{n=1}^{\infty} \frac{(-1)^{n} \cos \left(\Lambda_{s,(2)}^{n} \xi\right)}{(2 n+1)^{3}} e^{i \omega t} .
$$




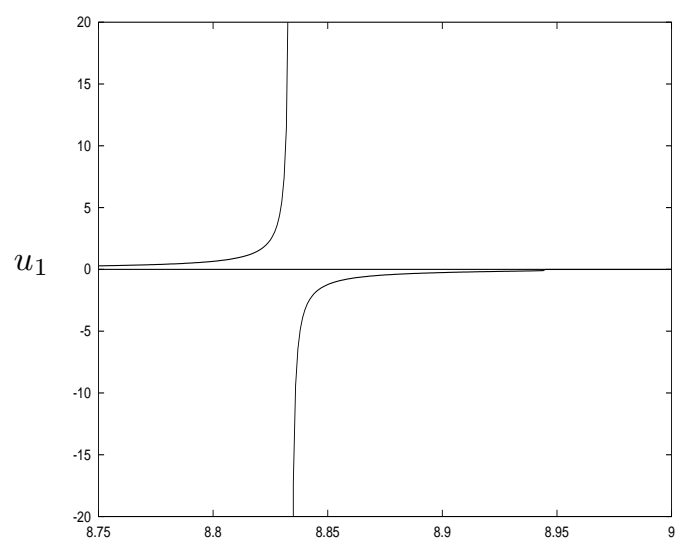

$(a)$

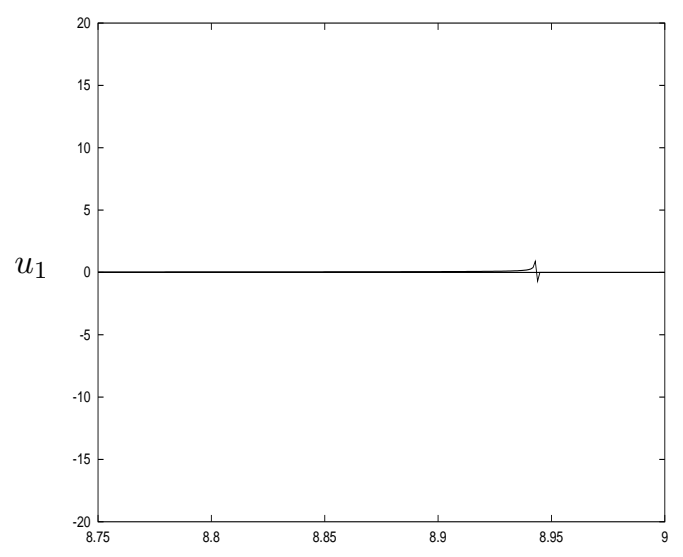

(c),

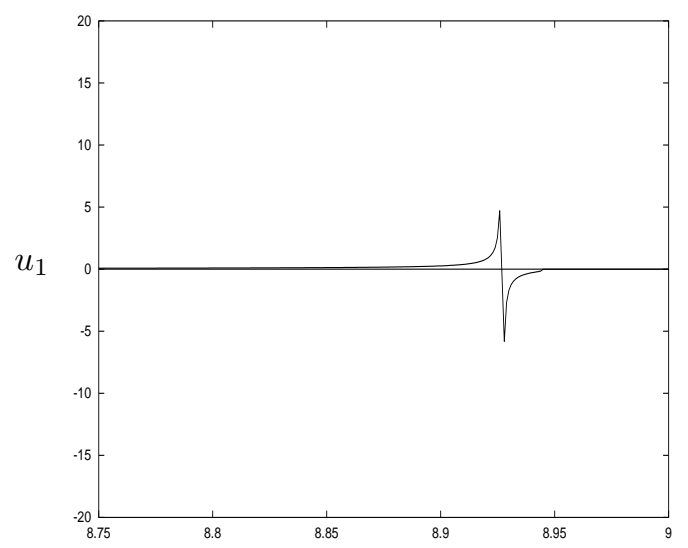

(b),

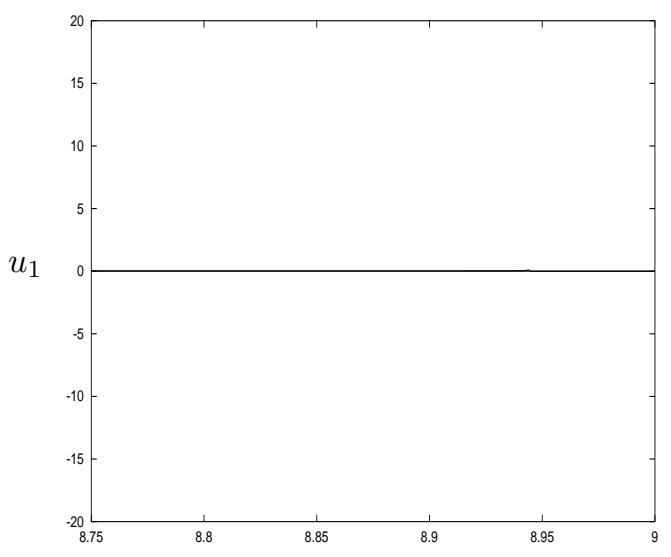

$(d)$,

$\Omega$

Figure 6: The displacement $u_{1}$ for different stress values as the surface wave speed degenerates into a body wave for the linear strain-energy function (19) with $\lambda_{1}=1.3, \lambda_{2}=0.7$ and $W_{1}^{*}=W_{2}^{*}=W_{3}^{*}=W_{4}^{*}=0.5$ and $\sigma_{3}=\gamma+\Delta$ for (a) $\Delta=1.5$, (b) $\Delta=1.0$, (c) $\Delta=0.5$, (d) $\Delta=0.1$.

The boundary conditions may then be represented in the form

$$
\begin{aligned}
& \sum_{n=1}^{\infty}\left\{\mathcal{T}_{1}\left(\hat{q}_{1}^{I I}\right) W_{n}^{(1)}+\mathcal{T}_{1}\left(\hat{q}_{2}^{I I}\right) W_{n}^{(2)}\right\}=4 \sigma_{0} h \sum_{n=1}^{\infty} \frac{(-1)^{n+1}}{\left[\Lambda_{s,(2)}^{n}\right]^{4}} \\
& \sum_{n=1}^{\infty}\left\{\mathcal{T}_{2}\left(\hat{q}_{1}^{I I}, \hat{\Omega}_{2}^{2}\right) W_{n}^{(1)}+\mathcal{T}_{2}\left(\hat{q}_{2}^{I I}, \hat{\Omega}_{2}^{2}\right) W_{n}^{(2)}\right\}=0
\end{aligned}
$$

from which the constants $W_{n}^{(1)}$ and $W_{n}^{(2)}$ may be expressed as

$$
W_{n}^{(1)}=\frac{4 \sigma_{0} h \mathcal{T}_{2}\left(\hat{q}_{2}^{I I}, \hat{\Omega}_{2}^{2}\right)(-1)^{n+1}}{\left[\Lambda_{s,(2)}^{n}\right]^{4} \mathcal{S}_{2 s}\left(\hat{q}_{1}^{I I}, \hat{q}_{2}^{I I}, \hat{\Omega}_{2}^{2}\right)}, \quad W_{n}^{(2)}=\frac{4 \sigma_{0} h \mathcal{T}_{2}\left(\hat{q}_{1}^{I I}, \hat{\Omega}_{2}^{2}\right)(-1)^{n}}{\left[\Lambda_{s,(2)}^{n}\right]^{4} \mathcal{S}_{2 s}\left(\hat{q}_{1}^{I I}, \hat{q}_{2}^{I I}, \hat{\Omega}_{2}^{2}\right)}
$$

Appropriate solutions for the displacement components and pressure increment, associated 
with the $n^{\text {th }}$ harmonic, are given by

$$
\begin{aligned}
& u_{1}^{(n)}=\left\{\sum_{m=1}^{2}-i \hat{q}_{m}^{I I} W_{n}^{(m)} e^{-\hat{q}_{m}^{I I} \Lambda_{s,(2)}^{n} \zeta} \cos \left(\Lambda_{s,(2)}^{n} \xi\right) e^{i \omega t},\right. \\
& u_{3}^{(n)}=\left\{\sum_{m=1}^{2} W_{n}^{(m)} e^{-i \hat{q}_{m}^{I I} \Lambda_{s,(2)}^{n} \zeta}\right\} \sin \left(\Lambda_{2}^{n} \xi\right) e^{i \omega t}, \\
& p^{*(n)}=\left\{\sum_{m=1}^{2}-i p_{2} \hat{q}_{m}^{I I} W_{n}^{(m)} \mathcal{H}\left(\hat{q}_{m}^{I I}, \hat{\Omega}_{2}^{2}\right) e^{-i \hat{q}_{m}^{I I} \Lambda_{s,(2)}^{n} \zeta}\right\} \sin \left(\Lambda_{s,(2)}^{n} \xi\right) e^{i \omega t} .
\end{aligned}
$$

\subsection{Edge vibration of a nearly inextensible semi-infinite strip}

We now examine the free and forced edge vibration problems in case of the fibres being almost inextensible. We consider the strain-energy function of the form given in (27). Let us now specify the fiber direction as $\phi=0$ and the propagation direction along $x_{1}$. The associated non-zero elasticity tensor components take form

$$
\begin{aligned}
& B_{1111}=\tilde{B}_{1111}+T_{0}+E, \quad B_{1133}=\tilde{B}_{1133}, \quad B_{1313}=\tilde{B}_{1313}+T_{0}, \\
& B_{1331}=\tilde{B}_{1331}, \quad B_{3131}=\tilde{B}_{3131}, \quad B_{3333}=\tilde{B}_{3333},
\end{aligned}
$$

and hence

$$
\alpha=\tilde{\alpha}+T_{0}, \quad \gamma=\tilde{\gamma}, \quad 2 \beta=2 \tilde{\beta}+T_{0}+E,
$$

where as previously the values with over-tilde denote their dependence only on the invariants $I_{1}, I_{2}$ and $I_{3}$.

This allows us to perform a simple analysis of (38), from which we deduce that

$$
\hat{q}_{1}^{2}+\hat{q}_{2}^{2}=\mathcal{O}(E), \quad \hat{q}_{1}^{2} \hat{q}_{2}^{2}=\mathcal{O}(1),
$$

which implies without loss of generality that

$$
\hat{q}_{1}^{2}=\mathcal{O}(E), \quad \hat{q}_{2}^{2}=\mathcal{O}\left(E^{-1}\right) .
$$

The free displacements and the pressure increment eigenmodes are provided by expressions (46) and (54) for the first and second families of natural frequencies, respectively. Let us now illustrate the dependence of the displacement eigenfunctions on the value of extensional modulus $E$ by considering the first family of solutions. It may be deduced from (46) that in case of large $E$ the displacements will not decay exponentially. However $u_{1}$ will tend to zero as $E$ tends to infinity. The dependence of the in-plane displacement on 


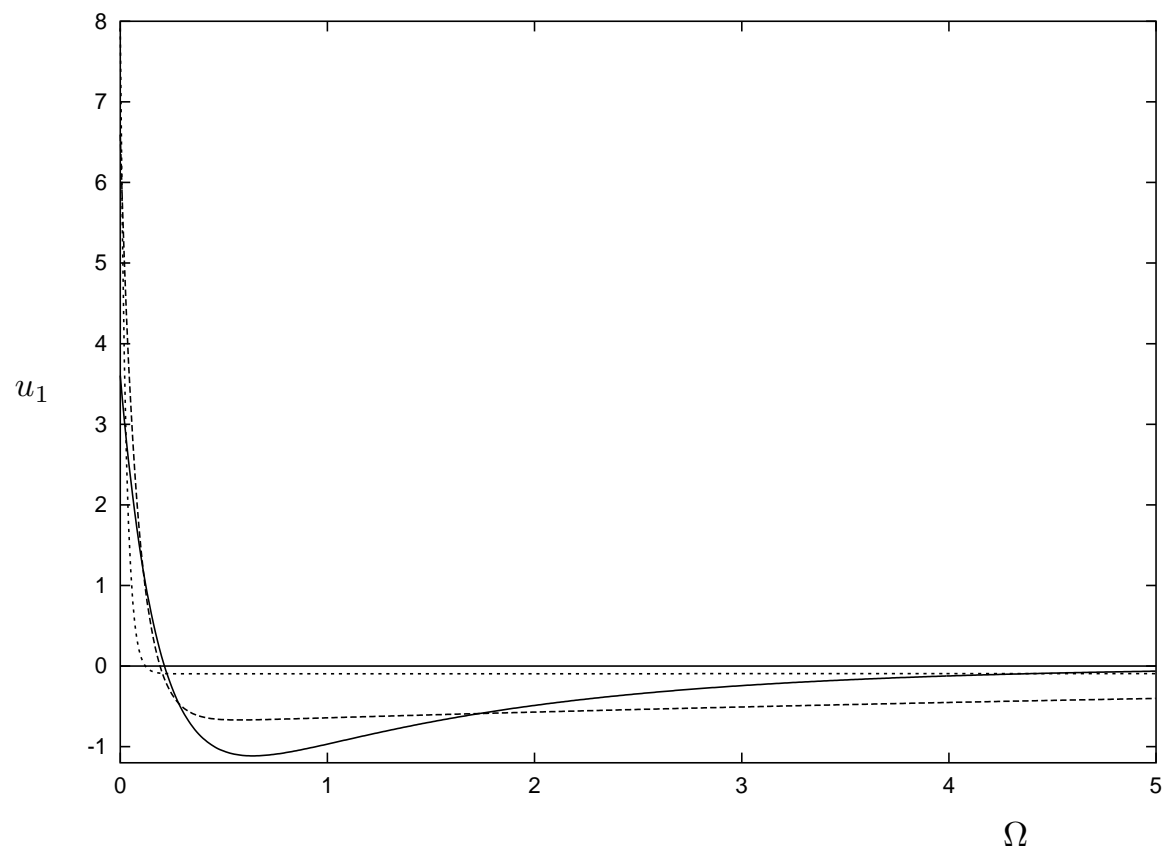

Figure 7: The displacement $u_{1}$ against the longitudinal variable $x_{3}$ for different values of extensional modulus in case of the strain-energy function (27) with $\lambda_{1}=1.3, \lambda_{2}=0.7$ and $W_{1}^{*}=W_{2}^{*}=W_{3}^{*}=W_{4}^{*}=0.5: E=10.0$ (solid curve), $E=100.0$ (dashed curve) and $E=1,000.0$ (dotted curve). 
the longitudinal variable $x_{3}$ is illustrated in Figure 7 for different values of the extensional modulus $E$. It seems natural from the corresponding illustrations for surface wave, in Figure 2, that as $E$ increases the localisation effect weakens up to its disappearance as the surface wave tends to a body wave. The confirmation of this may be observed from Figure 7. The in-plane displacement $u_{1}$ is small of order $\mathcal{O}\left(E^{-0.5}\right)$, whereas the other displacement $u_{3}$ is relatively large in comparison and is not localised near the edge due to the fact that it represents a body wave.

Let us now discuss briefly the forced vibration problem. The resonance amplitude is anticipated to vanish as $E$ tends to infinity due to the fact that a surface wave degenerates into a body wave. A numerical investigation quickly confirms this. We remark that the illustrations look very similar to those on Figure 6 and therefore are not presented in this paper.

\section{Conclusions}

The problem of surface wave propagation in pre-stressed, incompressible, transversely isotropic elastic media has been investigated, together with the closely related problem of localised edge vibration of a semi-infinite strip composed of similar material. It has been shown that there exists a very simple relationship between the edge vibration frequencies and the Rayleigh surface wave speed. For certain pre-stressed states the Rayleigh surface wave speed may approach either zero or the limiting body wave speed. These cases are associated with quasi-static surface deformations and degeneration of a surface wave into a body wave, respectively. Both of these types of pre-stressed state have been shown to greatly influence the edge spectrum, with the former yielding a large increase in spectrum density and the former removing the localised vibrations completely.

The study lays the foundation for more detailed investigations to fully elucidate the class of boundary conditions, material anisotropy and pre-stress which can support such vibration modes. As we have established in the case of pre-stress, the existence of such localised phenomena cannot be taken for granted. It is envisaged that having the ability to predict the existence of such localised phenomena will have application to non-destructive evaluation. Moreover, the specific application within thin structures has direct relevance to understanding the dynamic behaviour of thin structural components. This paper helps provide the basis for various examples of thin structures, many of which will require very 
delicate treatment of the boundary conditions.

\section{Acknowledgement}

The work of D.A. Prikazchikov was supported by a London Mathematical Society grant and also by the University of Salford. These awards are very gratefully acknowledged.

\section{References}

1. Lord Rayleigh, "On waves propagated along the plane surface of an elastic solid," Proc. Lond. Math. Soc., 17, 4-11 (1885).

2. M. A. Hayes, and R. S. Rivlin, "Surface waves in deformed elastic materials," Arch. Ration. Mech. Analysis, 8, 358-380 (1961).

3. P. Chadwick, and D. A. Jarvis, "Surface waves in a pre-stressed elastic body," Proc. Roy. Soc. Lond., Ser. A, 366, 517-536 (1979).

4. M. A. Dowaikh and R. W. Ogden, "On surface waves and deformations in a pre-stressed incompressible elastic solid," IMA Jl. Appl. Math., 44, 261-284 (1990).

5. E. A. Shaw "On the resonant vibrations of thick barium titanate disks," J. Acoust. Soc. Am., 28, 38-50 (1956).

6. D. C. Gazis and R. D. Mindlin, "Extensional vibrations and waves in a circular disk and a semi-infinite plate," J. Appl. Mech., 27, 541-547 (1960).

7. P. J. Torvik, "Reflection of wave trains in semi-infinite plates," J. Acoust. Soc. Am., 41, 346-353 (1967).

8. R. D. Gregory and I. Gladwell, "The reflection of a symmetric Rayleigh-Lamb wave at the fixed or free edge of a plate," J. Elasticity, 13, pp. 185-206 (1983).

9. I. Roitberg, D. Vassiliev and T. Weidl, "Edge resonances in an elastic semi-strip," Quart Jl. Mech. Appl. Math., 51, 1-13 (1998).

10. J. D. Kaplunov, L. Yu. Kossovich and M. V. Wilde, "Free localized vibrations of a semi-infinite cylindrical shell," J. Acoust. Soc. America 107, 1383-1393 (2000).

11. J. D. Kaplunov, D. A. Prikazchikov and G. A. Rogerson, "Edge vibration of a prestressed semi-infinite strip with traction-free edge and mixed face boundary conditions," Jl. Appl. Math. Phys. (ZAMP), 55, 701-719 (2004). 
12. J. D. Kaplunov, D. A. Prikazchikov and G. A. Rogerson, "On 3D edge waves in a semi-infinite plate," J. Acoust. Soc. America, 118(5), pp.2975-2983, 2005.

13. D. A. Prikazchikov and G. A. Rogerson, "Some comments on the dynamic properties of anisotropic and strongly anisotropic pre-stressed elastic solids," Int. Jl. Eng. Sci., 41, 149-171, (2003).

14. R. W. Ogden, Non-linear elastic deformations. Dover: Ellis Horwood (1984).

15. G.A. Rogerson and N. H. Scott, "Wave propagation in singly constrained and nearly constrained elastic materials," Quart Jl. Mech. Appl. Math., 45, 77-99 (1992).

16. N. H. Scott, "Waves in homogeneously pre-strained incompressible, almost inextensible, fibre-reinforced elastic material," Proc. Roy. Irish Acad., 92, pp. 9-36 (1992).

17. D. A. Prikazchikov and G. A. Rogerson, "On surface wave propagation in incompressible, transversely isotropic, pre-stressed elastic half-spaces". Int. Jl. Eng. Sci., 42, pp. 967-986 (2004).

18. A. J. M. Spencer, Deformations of fibre-reinforced materials. Clarendon Press, Oxford (1972).

19. Oppenheimer, M, "Global warming and the stability of the West Antarctic Ice Sheet," Nature, 393, p 325 (1998).

20. Inoue, Y, Arai, M, Tabeta, S and Nakazawa, K, "Dynamic Behaviour of a Floating Airport and Its Effects on Ocean Current",Proc. International Offshore and Polar Engineering Conference, 3, pp406-413 (1995).

21. Suzuki,H. and Yoshida,K. "A Consideration on the Dynamic Behaviour and the Structural Design of Large Scale Floating Structure", Journal of the Society of Naval Architects of Japan, 178, pp.473-483 (1995).

\section{Appendix A}

The components of the fourth order elasticity tensor are given by

$$
B_{\text {milk }}=F_{m A} F_{i B} \frac{\partial^{2} W^{*}}{\partial F_{k B} \partial F_{i A}}
$$

and evaluated in the pre-stressed equilibrium state. The specific components of this tensor are obtained by inserting the strain energy function (2.2) into the above definition, with 
the non-zero components taking the following forms

$$
\begin{aligned}
& B_{1111}=2 \lambda_{1}^{2}\left[W_{1}+W_{2} \Sigma_{23}\right]+4 \lambda_{1}^{4}\left[W_{11}+2 W_{12} \Sigma_{23}+W_{22} \Sigma_{23}^{2}\right] \\
& +4 \lambda_{1}^{2} c_{\phi}^{2}\left[3 W_{3}+4 \lambda_{1}^{2}\left\{W_{31}+W_{32} \Sigma_{23}+W_{33} c_{\phi}^{2}\right\}+4 W_{34} c_{\phi}^{2}\right] \\
& +2 c_{\phi}^{2}\left[W_{4}+2 W_{44} c_{\phi}^{2}+4 \lambda_{1}^{2}\left\{W_{41}+W_{42} \Sigma_{23}\right\}\right], \\
& B_{1113}=2 s_{2 \phi}\left[\lambda_{1}^{2}\left\{W_{3}+\Sigma_{13}\left(W_{31}+W_{32} \Sigma_{23}+2 W_{33} c_{\phi}^{2}\right)+W_{41}+W_{42} \Sigma_{23}\right\}\right. \\
& \left.+c_{\phi}^{2}\left(W_{34}\left(2 \lambda_{1}^{2}+\Sigma_{13}\right)+W_{44}\right)\right], \\
& B_{1131}=s_{2 \phi}\left[\left(2 \lambda_{1}^{2}+\Sigma_{13}\right)\left(W_{3}+2 W_{34} c_{\phi}^{2}\right)+2 \lambda_{1}^{2}\left(W_{41}+W_{42} \Sigma_{23}\right)+W_{4}+2 W_{44} c_{\phi}^{2}\right. \\
& \left.+2 \lambda_{1}^{2} \Sigma_{13}\left\{W_{31}+W_{32} \Sigma_{23}+2 W_{33} c_{\phi}^{2}\right\}\right], \\
& B_{1133}=4 \lambda_{1}^{2} \lambda_{3}^{2}\left[W_{2}+W_{11}+W_{12}\left(\Sigma_{12}+\Sigma_{23}\right)+W_{22} \Sigma_{12} \Sigma_{23}\right]+4 W_{41}\left(\lambda_{1}^{2} s_{\phi}^{2}+\lambda_{3}^{2} c_{\phi}^{2}\right) \\
& +8 \lambda_{1}^{2} \lambda_{3}^{2}\left[W_{31}+W_{32}\left(\Sigma_{12} c_{\phi}^{2}+\Sigma_{23} s_{\phi}^{2}\right)\right]+s_{2 \phi}^{2}\left[W_{44}+2 W_{34} \Sigma_{13}+4 W_{33} \lambda_{1}^{2} \lambda_{3}^{2}\right] \\
& +4 W_{42}\left[\lambda_{1}^{2} \lambda_{3}^{2}+\lambda_{2}^{2}\left(\lambda_{1}^{2} s_{\phi}^{2}+\lambda_{3}^{2} c_{\phi}^{2}\right)\right], \\
& B_{1313}=2 \lambda_{1}^{2}\left(W_{1}+W_{2} \lambda_{2}^{2}\right)+2 W_{3}\left(\lambda_{1}^{2}+\Sigma_{13} c_{\phi}^{2}\right)+2 W_{4} c_{\phi}^{2}+s_{2 \phi}^{2}\left[W_{33} \Sigma_{13}^{2}+2 W_{34} \Sigma_{13}+W_{44}\right] \text {, } \\
& B_{1331}=-2 W_{2} \lambda_{1}^{2} \lambda_{3}^{2}+2 W_{3}\left(\lambda_{1}^{2} s_{\phi}^{2}+\lambda_{3}^{2} c_{\phi}^{2}\right)+s_{2 \phi}^{2}\left[W_{33} \Sigma_{13}^{2}+2 W_{34} \Sigma_{13}+W_{44}\right], \\
& B_{1333}=s_{2 \phi}\left[\left(\Sigma_{13}+2 \lambda_{3}^{2}\right)\left(W_{3}+2 W_{34} s_{\phi}^{2}\right)+W_{4}+2 W_{44} s_{\phi}^{2}\right. \\
& \left.+2 \lambda_{3}^{2}\left\{\Sigma_{13}\left(W_{31}+2 W_{33} s_{\phi}^{2}\right)+\Sigma_{12}\left(W_{42}+W_{32} \Sigma_{13}\right)\right\}\right], \\
& B_{3131}=2 \lambda_{3}^{2}\left(W_{1}+W_{2} \lambda_{2}^{2}\right)+2 W_{3}\left(\lambda_{3}^{2}+\Sigma_{13} s_{\phi}^{2}\right)+2 W_{4} s_{\phi}^{2} \\
& +s_{2 \phi}^{2}\left[W_{44}+2 W_{34} \Sigma_{13}+W_{33} \Sigma_{13}^{2}\right], \\
& B_{3133}=2 \lambda_{3}^{2} s_{2 \phi}\left[W_{3}+W_{41}+W_{42} \Sigma_{12}+\Sigma_{13}\left\{W_{33} s_{\phi}^{2}+W_{32} \Sigma_{12}\right\}\right] \\
& +2 s_{\phi}^{2} s_{2 \phi}\left[W_{44}+W_{34}\left(\Sigma_{13}+2 \lambda_{3}^{2}\right)\right], \\
& B_{3333}=2 \lambda_{3}^{2}\left[W_{1}+W_{2} \Sigma_{12}\right]+4 \lambda_{3}^{4}\left[W_{11}+2 W_{12} \Sigma_{12}+W_{22} \Sigma_{12}^{2}\right] \\
& +4 \lambda_{3}^{2} s_{\phi}^{2}\left[3 W_{3}+4 \lambda_{3}^{2}\left\{W_{31}+W_{32} \Sigma_{12}+W_{33} s_{\phi}^{2}\right\}+4 W_{34} s_{\phi}^{2}\right] \\
& +2 s_{\phi}^{2}\left[W_{4}+2 W_{44} s_{\phi}^{2}+4 \lambda_{3}^{2}\left\{W_{41}+W_{42} \Sigma_{12}\right\}\right],
\end{aligned}
$$

where

$$
\begin{gathered}
W_{k}=\frac{\partial W}{\partial I_{k}}, \quad W_{n m}=\frac{\partial^{2} W}{\partial I_{n} \partial I_{m}}, \quad k, m, n=1,2,3,4, \\
c_{\phi}=\cos \phi, \quad s_{\phi}=\sin \phi, \quad s_{2 \phi}=\sin 2 \phi, \quad \Sigma_{i j}=\lambda_{i}^{2}+\lambda_{j}^{2}, \quad 1 \leq i \neq j \leq 3 .
\end{gathered}
$$

